\title{
A novel HIV-1 protease inhibitor, GRL-044, has potent activity against various HIV-1s with an extremely high genetic barrier to the emergence of HIV-1 drug resistance
}

\author{
Manabu Aoki ${ }^{1,2}$, Simon B. Chang', Debananda Das', Cuthbert Martyr ${ }^{3}$, Nicole S. Delino', Yuki Takamatsu', \\ Arun K. Ghosh ${ }^{3}$, Hiroaki Mitsuya ${ }^{1,2,4, *}$ \\ ${ }^{1}$ Experimental Retrovirology Section, HIV and AIDS Malignancy Branch, National Cancer Institute, National Institutes of Health, Bethesda, MD, USA; \\ ${ }^{2}$ Department of Refractory Viral Infections, National Center for Global Health and Medicine Research Institute, Tokyo, Japan; \\ ${ }^{3}$ Departments of Chemistry and Medicinal Chemistry, Purdue University, West Lafayette, IN, USA; \\ ${ }^{4}$ Deprtment of Clinical Sciences, Kumamoto University Hospital, Kumamoto, Japan.
}

\begin{abstract}
We designed, synthesized, and identified two novel nonpeptidic HIV-1 protease inhibitors (PIs), GRL037 and GRL-044, containing P2-tetrahydropyrano-tetrahydrofuran (Tp-THF), P1-benzene and P1-methoxybenzene, respectively, and P2'-isopropyl-aminobenzothiazole (Ip-Abt), based on the structure of the prototypic PI, darunavir (DRV). The $50 \%$ inhibitory concentrations $\left(\mathrm{IC}_{50} \mathrm{~s}\right)$ of GRL-037 and GRL-044 against wild-type HIV- $1_{\text {NL4-3 }}$ were 0.042 and $0.0028-0.0033 \mathrm{nM}$ with minimal cytotoxicity profiles compared to the $\mathrm{IC}_{50}$ values of four most potent FDAapproved PIs, ranging from 2.6 to $70 \mathrm{nM}$. GRL-044 was also potent against $\mathrm{HIV}-2_{\mathrm{EHO}}\left(\mathrm{IC}_{50}=0.0004 \mathrm{nM}\right)$ and various PI-resistant $\mathrm{HIV}-1$ variants $\left(\mathrm{IC}_{50}\right.$ ranging from 0.065 to $\left.19 \mathrm{nM}\right)$. In the selection assays we conducted, the emergence of HIV-1 variants resistant to GRL-044 was significantly delayed compared to that against DRV. Thermal stability test using differential scanning fluorimetry employing purified HIV-1 protease (PR) and SYPRO ${ }^{\circledR}$ Orange showed that both GRL-037 and GRL-044 tightly bound to PR. A28S substitution emerged in the homologous recombinationbased selection assays with GRL-044. Structural analyses showed that the larger size of GRL-044 over DRV, enabling GRL-044 to fit better to the hydrophobic cavity of protease, contributed to the greater potency of GRL044 against HIV-1. Structural analyses also suggested that the van der Waals surface contact of GRL-044 with A28' appears to be better compared to that of DRV because of the larger surface of Ip-Abt of GRL-044, which may be partially responsible for the emergence of A28S. The present antiviral data and structural features of GRL-044 should provide molecular insights for further design and development of potent and "resistance-repellant" novel PIs.
\end{abstract}

Keywords: Protease inhibitor, HIV-1, AIDS, drug resistance

\section{Introduction}

Combined antiretroviral therapy or cART for HIV1 infection and AIDS has dramatically changed the clinical profiles and prognosis of HIV-1 infection/ AIDS and significantly extended the life expectancy of people with HIV-1 infection/AIDS. In fact, cART has been definitely shown to reduce HIV-1 transmission at very high rates (1). The most recently completed 7-year PARTNER2 study has revealed no cases of HIV transmission among $\sim 800$ sero-discordant gay male couples, who engaged in condomless anal sex a total of 76,088 times while the HIV-1-infected partners were virally suppressed on cART. The study supports the message of the $\mathrm{U}=\mathrm{U}$ (undetectable=untransmittable) campaign and the benefits of early testing and cART (2). Nevertheless, individuals who initially attain complete suppression of HIV-1 to undetectable levels may suffer treatment failure because of the flaws of cART such as inherent adverse effects and the propensity of HIV1 to develop drug resistance. Thus, development of therapeutics for HIV-1 infection/AIDS that are more potent, less or least toxic, and do not permit or delay the emergence of drug resistance with more favorable dosing regimen capabilities is critically important (3-6).

In the present work, we designed, synthesized, and identified two novel nonpeptidic HIV-1 protease inhibitors (PIs), GRL-037 and GRL-044, containing (i) P2-tetrahydropyrano-tetrahydrofuran (Tp-THF), (ii) P1-benzene and P1-methoxybenzene, respectively, and (iii) P2'-isopropyl-aminobenzothiazole (Ip-Abt) on the structure of darunavir (DRV) $(7,8)$, which is the last approved PI for the treatment of HIV-1 infection/AIDS and has been most used worldwide $(8-10)$. Both GRL- 
037 and GRL-044 showed potent antiviral activity against wild-type HIV-1, but GRL-044, in particular, exerted potent antiviral activity against wild-type HIV1, HIV-2, and various drug-resistant HIV-1 variants. Structural analyses showed that the larger and "right" size of GRL-044 over DRV contributed to the greater potency of GRL-044 against HIV-1. The present data suggest that the combination of the three moieties in GRL-044 resulted in highly potent activity against wild-type and multi-PI-resistant HIV-1 strains and contributed toward its extremely high genetic barrier to the emergence of HIV-1 variants to GRL-044 compared to its prototypic PI, DRV. The antiviral properties and structural features of GRL-044 should shed lights in the design and development of more potent and "resistancerepellant" PIs with novel structural features.

\section{Materials and Methods}

\section{Antiviral agents}

Two nonpeptidic PIs, GRL-0476 and GRL-1398, and their properties were previously published $(11,12)$, while GRL-037 and GRL-044 were newly designed and synthesized. The method of synthesis of GRL-037 and GRL-044 will be published elsewhere by A. K. Ghosh et al. DRV was synthesized as previously described (7). Amprenavir (APV), lopinavir (LPV), atazanavir (ATV), saquinavir (SQV), and zidovudine (AZT) were purchased from Sigma-Aldrich (St. Louis, MO).

\section{Cells and viruses}

MT-4 cells were grown in RPMI 1640-based culture medium supplemented with $10 \%$ fetal bovine serum (BenchMark $^{\mathrm{TM}}$ Fetal Bovine Serum, Gemini BioProducts) plus $50 \mathrm{U}$ of penicillin and $50 \mu \mathrm{g}$ of kanamycin per $\mathrm{mL}$. The following HIV-1 strains were used for the drug susceptibility assay and in vitro selection experiments: eleven HIV-1 clinical strains $\left(\mathrm{HIV}_{\mathrm{A}}, \mathrm{HIV}_{\mathrm{B}}, \mathrm{HIV}_{\mathrm{C}}, \mathrm{HIV}_{\mathrm{G}}, \mathrm{HIV}_{\mathrm{TM}}, \mathrm{HIV}_{\mathrm{MM}}, \mathrm{HIV}_{\mathrm{JSL}}\right.$, $\mathrm{HIV}_{\mathrm{SS}}, \mathrm{HIV}_{\mathrm{ES}}, \mathrm{HIV}_{\mathrm{EV}}$, and $\mathrm{HIV}_{13-52}$ ), which were originally isolated from patients with AIDS who were enrolled in an open-labeled clinical study of APV and abacavir at the Clinical Center, National Institutes of Health, were randomly chosen from the enrollees, who had failed APV-plus-ABC therapy in a phase I/II study of tenofovir disoproxil fumarate $(13,14)$. Such patients had failed existing anti-HIV-1 regimens after receiving 7 to 11 anti-HIV-1 drugs over the previous 24 to 83 months in late 1990s. Such clinical strains contained 8 to 16 amino acid substitutions in the protease-encoding region (Figure 4), which are associated with HIV-1 resistance to various PIs and have been genotypically and phenotypically characterized to be multi-protease inhibitor (PI)-resistant HIV-1 (15). Five HIV-1 variants resistant to PI that had been selected in vitro with each of four FDA-approved PIs (DRV, APV, ATV, and LPV) were also employed $(16,17)$. All the variants used in the present study were confirmed to have acquired multiple amino acid substitutions in protease (see Table 1 footnote), which have reportedly been associated with viral resistance to PIs. Each of the PI-selected

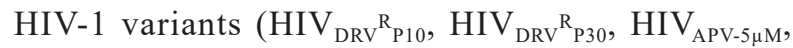
$\mathrm{HIV}_{\mathrm{ATV}-5 \mu \mathrm{M}}$, and $\left.\mathrm{HIV}_{\mathrm{LPV}-5 \mu \mathrm{M}}\right)$ was highly resistant to the corresponding PI, with which the variant was selected, and the differences in the $\mathrm{IC}_{50} \mathrm{~s}$ relative to the $\mathrm{IC}_{50}$ of each drug against wild-type HIV-1 (HIV-1 $\left.{ }_{\mathrm{NL} 4-3}\right)$ ranged from $>14$ to $>303$-fold (Table 1). All the HIV-1 strains used in this study were stored at $-80^{\circ} \mathrm{C}$ until use.

\section{Antiviral and cytotoxicity assays}

Antiviral assays were conducted as previously described (14). Briefly, the designated concentrations of each compound tested were prepared by ten-fold serial

Table 1. Antiviral activity of GRL-037 and GRL-044 against in vitro PI-selected HIV-1 variants and multi-drug resistant HIV-1 clinical isolates

\begin{tabular}{|c|c|c|c|c|c|c|c|c|}
\hline \multirow{2}{*}{ Virus } & \multicolumn{8}{|c|}{ Mean $\mathrm{IC}_{50}(\mathrm{nM}) \pm \mathrm{SD}$} \\
\hline & APV & LPV & ATV & DRV & GRL-0476 & GRL-1398 & GRL-037 & GRL-044 \\
\hline $\mathrm{HIV}-1_{\mathrm{NL} 4-3}$ & $70 \pm 41$ & $57 \pm 3$ & $3.3 \pm 0.5$ & $3.5 \pm 0.7$ & $4.8 \pm 1.0$ & $0.29 \pm 0.05$ & $0.042 \pm 0.012$ & $0.0033 \pm 0.0024$ \\
\hline $\mathrm{HIV}_{\mathrm{APV}-5 \mu \mathrm{M}^{\mathrm{a}}}$ & $>1,000(>14)$ & $390 \pm 62(6.8)$ & $4.5 \pm 0.3(1.4)$ & $336 \pm 65(96)$ & $\mathrm{ND}^{\mathrm{c}}$ & $\mathrm{ND}^{\mathrm{c}}$ & $47 \pm 5(1,119)$ & $4.5 \pm 0.2(1,364)$ \\
\hline $\mathrm{HIV}_{\mathrm{ATV}-5 \mu \mathrm{M}^{\mathrm{a}}}$ & $>1,000(>14)$ & $378 \pm 70(6.6)$ & $>1,000(>303)$ & $13 \pm 4(3.7)$ & $\mathrm{ND}^{\mathrm{c}}$ & $\mathrm{ND}^{\mathrm{c}}$ & $0.44 \pm 0.12(10)$ & $0.065 \pm 0.024(20)$ \\
\hline $\mathrm{HIV}_{\mathrm{LPV}-5 \mu \mathrm{M}^{\mathrm{a}}}$ & $>1,000(>14)$ & $>1,000(>18)$ & $503 \pm 31(152)$ & $409 \pm 23(117)$ & $\mathrm{ND}^{\mathrm{c}}$ & $\mathrm{ND}^{\mathrm{c}}$ & $40 \pm 3(952)$ & $3.8 \pm 0.3(1,152)$ \\
\hline $\mathrm{HIV}_{\mathrm{DRV}}{ }_{\mathrm{P} 10^{\mathrm{a}}}^{\mathrm{a}}$ & $>1,000(>14)$ & $>1,000(>18)$ & $>1,000(>303)$ & $140 \pm 41(40)$ & $382 \pm 55(80)$ & $45 \pm 7(154)$ & $23 \pm 4(548)$ & $3.3 \pm 0.2(1,000)$ \\
\hline $\mathrm{HIV}_{\mathrm{DRV}}{ }^{\mathrm{R}}{ }_{\mathrm{P} 30}^{\mathrm{a}}$ & $>1,000(>14)$ & $>1,000(>18)$ & $>1,000(>303)$ & $465 \pm 107(133)$ & $393 \pm 61(82)$ & $173 \pm 29(598)$ & $83 \pm 18(1,976)$ & $19 \pm 2(5,758)$ \\
\hline $\mathrm{HIV}_{\mathrm{B}}^{\mathrm{b}}$ & $>1,000(>14)$ & $>1,000(>18)$ & $>1,000(>303)$ & $40 \pm 8(11)$ & $\mathrm{ND}^{\mathrm{c}}$ & $\mathrm{ND}^{\mathrm{c}}$ & $46 \pm 5(1,095)$ & $4.6 \pm 0.4(1,394)$ \\
\hline $\mathrm{HIV}_{\mathrm{C}}^{\mathrm{b}}$ & $>1,000(>14)$ & $>1,000(>18)$ & $418 \pm 49(127)$ & $38 \pm 2(11)$ & $\mathrm{ND}^{\mathrm{c}}$ & $\mathrm{ND}^{\mathrm{c}}$ & $5.5 \pm 0.3(131)$ & $2.6 \pm 0.8(788)$ \\
\hline
\end{tabular}

The amino acid substitutions identified in protease of $\mathrm{HIV}_{\mathrm{APV}-5 \mathrm{M}}, \mathrm{HIV}_{\mathrm{TV}-5 \mathrm{M}}, \mathrm{HIV}_{\mathrm{LPV}-5 \mu \mathrm{M}}, \mathrm{HIV}_{\mathrm{DRV}} \mathrm{R}_{\mathrm{P} 10}, \mathrm{HIV}_{\mathrm{DRV}}{ }^{\mathrm{P} 30}, \mathrm{HIV}_{\mathrm{B}}$, and $\mathrm{HIV}_{\mathrm{C}}$, compared to the wild-type HIV-1 ${ }_{\mathrm{NL4-3}}$ include L10F/V32I/L33F/M46L/I54M/A71V, L23I/E34Q/K43I/M46I/I50L/G51A/L63P/A71V/V82A/T91A, L10F/V32I/M46I/ I47A/A71V/I84V, L10I/ I15V/K20R/L24I/V32I/M36I/M46L/L63P/K70Q/V82A/L89M, L10I/I15V/K20R/L24I/V32I/M36I/M46L/L63P/K70Q/ V82A/I84V/L89M,L10I/L33I/M36I/M46I/ F53L/K55R/I62V/L63P/A71V/G73S/V82A/L90M/I93L, and L10I/I15V/K20R/L24I/M36I/M46L/I54V/

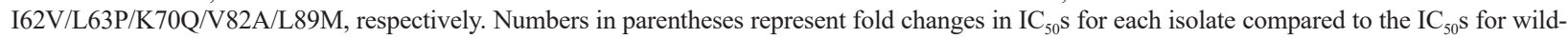
type HIV-1 $1_{\mathrm{NL} 4-3}$. All assays were conducted in triplicate, and the data shown represent mean values ( \pm 1 standard deviation) derived from the results of three independent experiments. ${ }^{a}$ Laboratory-selected drug-resistant HIV-1 variants; ${ }^{b}$ multidrug-resistant HIV-1 clinical isolates; ${ }^{c} \mathrm{ND}$ : not determined 
dilution using the working solution of the compound $(2 \mu \mathrm{M})$ directly in 96-well microtiter culture plates. MT-4 cells $(105 / \mathrm{mL})$ were exposed to fifty $50 \%$ tissue culture infective dose $\left(\right.$ TCID $\left._{50}\right)$ of each HIV-1 strain in the presence or absence of various concentrations of the compound and cultured at $37^{\circ} \mathrm{C}$. On the day 7 of culture, the supernatant was harvested and the amount of p24 Gag protein was determined using the fully automated chemiluminescent enzyme immunoassay system (Lumipulse G1200; Fujirebio Inc., Tokyo, Japan). The drug concentrations that suppressed the production of p24 Gag protein by 50\% (50\% inhibitory concentrations; $\mathrm{IC}_{50}$ ) were determined by comparison with the level of p24 production in drug-free control cell cultures. All assays were performed in triplicate. To determine the

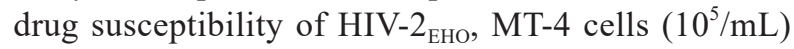
were exposed to $100 \mathrm{TCID}_{50}$ of the strain in the presence or absence of various concentrations of each compound, followed by cultivation at $37^{\circ} \mathrm{C}$ for 7 days. The number of viable cells was determined using the Cell Counting Kit-8 (Dojindo, Kumamoto, Japan) and the magnitude of viral inhibition by each compound was determined based on their inhibitory effects of the virally-induced cytopathicity in MT-4 cells. For cytotoxicity, cells were plated in 96-well microtiter culture plates at a density of $10^{5} / \mathrm{mL}$ and continuously exposed to various concentrations of each compound throughout the entire period of the culture. The number of viable cells in each well was determined using Cell Counting Kit-8. The $50 \%$ cytotoxic concentrations $\left(\mathrm{CC}_{50}\right)$ were determined as the concentration required to reduce the number of the cells by $50 \%$ compared to that of drug-unexposed control cultures.

In vitro generation of highly GRL-044-resistant HIV-1 variants

We attempted to select HIV-1 variants resistant against GRL-037 and GRL-044 as previously described (15). Briefly, thirty TCID50 of each of eleven HIV-1 clinical strains was mixed and propagated in a mixture of an equal number of phytohemagglutinin (PHA)-stimulated peripheral blood mononuclear cells $(\mathrm{PBMCs})\left(5 \times 10^{5}\right)$ and MT-4 cells $\left(5 \times 10^{5}\right)$, in an attempt to adapt the mixed viral population for their replication in MT-4 cells. The cell-free supernatant harvested on day 7 of the co-culture was referred to as $\mathrm{HIV}_{11 \mathrm{MIX}}$. In the first passage, MT- 4 cells $\left(5 \times 10^{5}\right)$ were exposed to the $\mathrm{HIV}_{11 \mathrm{MIX}}$-containing supernatants and cultured in the presence of each compound at an initial concentration of an $\mathrm{IC}_{50}$ dose. On the last day of each passage (weeks 1 to 3 ), $1.5 \mathrm{~mL}$ of the cell-free supernatant was harvested and transferred to a culture of fresh uninfected MT-4 cells in the presence of increased concentrations of the compound for the following round of culture. In this following round of culture, three drug concentrations (increased by one-, two-, and three- fold compared to the previous concentration) were employed. When the culture supernatant contained $>$ $200 \mathrm{ng} / \mathrm{mL}$ of p24 Gag protein, the HIV-1 isolate was assumed to have substantially replicated and the highest drug concentration among the three concentrations was used to continue the selection (for the next round of culture). This protocol was repetitively used until the drug concentration reached the targeted concentration (regularly $5 \mu \mathrm{M}$ ). Proviral DNA preparations obtained from the lysates of infected cells at indicated passages were subjected to nucleotide sequencing.

\section{Determination of nucleotide sequences}

Molecular cloning and determination of the nucleotide sequences of HIV-1 strains passaged in the presence of each compound were performed as previously described (16). In brief, high-molecular-weight DNA was extracted from HIV-1-infected MT-4 cells by using the InstaGene Matrix (Bio-Rad Laboratories, Hercules, CA) and was subjected to molecular cloning, followed by nucleotide sequence determination. The PCR primers used for the protease-encoding region were KAPA-1 (5'-GCA GGG CCC CTA GGA AAA AGG GCT GTT GG-3') and Ksma2.1 (5'-CCA TCC CGG GCT TTA ATT TTA CTG GTA C-3'). The PCR mixture consisted of $1 \mu \mathrm{L}$ proviral DNA solution, $10 \mu \mathrm{L}$ Premix Taq (Ex Taq Version; Takara Bio Inc., Otsu, Japan), and $10 \mathrm{pmol}$ of each PCR primer in a total volume of $20 \mu \mathrm{L}$. The PCR conditions used were an initial $1 \mathrm{~min}$ at $95^{\circ} \mathrm{C}$, followed by 30 cycles of $30 \mathrm{sec}$ at $95^{\circ} \mathrm{C}, 20$ sec at $55^{\circ} \mathrm{C}$, and $1 \mathrm{~min}$ at $72^{\circ} \mathrm{C}$, with a final $10 \mathrm{~min}$ of extension at $72^{\circ} \mathrm{C}$. The PCR products were purified using spin columns (illustra MicroSpin S-400 HR columns; GE Healthcare Life Science., Pittsburgh, PA), cloned directly, and subjected to sequencing using the BigDye Terminator v1.1 cycle sequencing kit (Applied Biosystems). The Sanger sequencing was conducted at the CCR Genomics Core of the National Cancer Institute.

\section{Thermal stability analysis using differential scanning} fluorimetry (DSF)

Wild-type HIV-1 protease (PR ${ }^{\mathrm{WT}}$ ) was purchased from ProSpec-Tany TechnoGene (Ness-Ziona, Israel). The final concentrations of each protein and compounds were $5 \mu \mathrm{M}$. Differential scanning fluorimetry (DSF) assays were conducted as previously described $(18,19)$, using a Protein Thermal Shift dye kit (Thermo Fisher Scientific) according to the manufacturer's instructions. All of the preparation procedures were conducted on ice and samples were kept on ice until the instrument run. Twenty microliters of $\mathrm{PR}^{\mathrm{WT}}$ was successively heated from 25 to $95^{\circ} \mathrm{C}$, and the changes in the fluorescence intensity were measured by using a StepOne realtime PCR system (Thermo Fisher Scientific). The data 
were analyzed using StepOne software version 2.3 and Protein Thermal Shift software version 1.0 (Thermo Fisher Scientific) and melting temperature $\left(T_{m}\right)$ values were obtained. All of the assays were conducted in duplicate on two different occasions.

Structural Analyses of GRL-044 Interactions with Wildtype HIV-1 Protease.

We started from the X-ray crystal structure of GRL142 complexed with wild-type PR ( $\mathrm{PR}^{\mathrm{WT}}$ ) (PDB ID: 5TYS). An initial conformation of the Tp-THF moiety was built by taking the coordinates from the conformation of the Tp-THF moiety of GRL-015 (PDB ID: 5CON (11)). The conformation of GRL142 was used to build the other moieties of GRL044. The assembled structure was suitably modified to build an initial conformation of GRL-044. GRL044 built in the active site of wild type protease was fully minimized using OPLS3 force field and used for subsequent analyses. Hydrogens were added to the crystal structure of DRV in complex with $\mathrm{PR}^{\mathrm{WT}}$ (PDB ID 4HLA (20)), protonation states of aspartates were assigned. A full minimization using OPLS3 force field was carried out. A cut-off distance of $3.1 \AA$ between a polar hydrogen and an oxygen or nitrogen atom, a minimum donor angle of $60^{\circ}$ between D-H-A, and a minimum acceptor angle of $90^{\circ}$ between $\mathrm{H}-\mathrm{A}-\mathrm{B}$ were used to define the presence of hydrogen bonds $(\mathrm{D}$,
$\mathrm{A}$, and $\mathrm{B}$ are donor, acceptor, and atom connected to acceptor, respectively). Connolly molecular surfaces for the inhibitors and selected PR residues from the active site were generated using a water sphere with a radius of $1.4 \AA$ as a probe. Software tools from Schrödinger, LLC, New York, NY, as implemented in the Maestro interface (version 10.7.015, Release 2016-3), were used for model building, visualization, and analysis.

\section{Results}

GRL-044 potently inhibits the replication of wild-type HIV-1

Based on the structure of DRV (8), we designed, synthesized, and identified two novel nonpeptidic PIs, GRL-037 and GRL-044, which contain P2tetrahydropyrano-tetrahydrofuran (Tp-THF), P1benzene and $\mathrm{P} 1$-methoxybenzene, respectively, and P2'-isopropyl-aminobenzothiazole (Ip-Abt) (Figure 1). Two previously reported PIs, GRL-0476 (11) and GRL1398 (12), both of which served as controls, and GRL037 and GRL-044 were all active against the wildtype HIV-1 strain (HIV-1 $1_{\mathrm{NL} 4-3}$ ) with $50 \%$ inhibitory concentration $\left(\mathrm{IC}_{50}\right)$ values of $4.8,0.29,0.042$, and $0.0033 \mathrm{nM}$, respectively in the drug susceptibility assays. Amprenavir (APV) and lopinavir (LPV) were much less active $\left(\mathrm{IC}_{50}=70\right.$ and $57 \mathrm{nM}$, respectively) and atazanavir (ATV) and darunavir (DRV) were
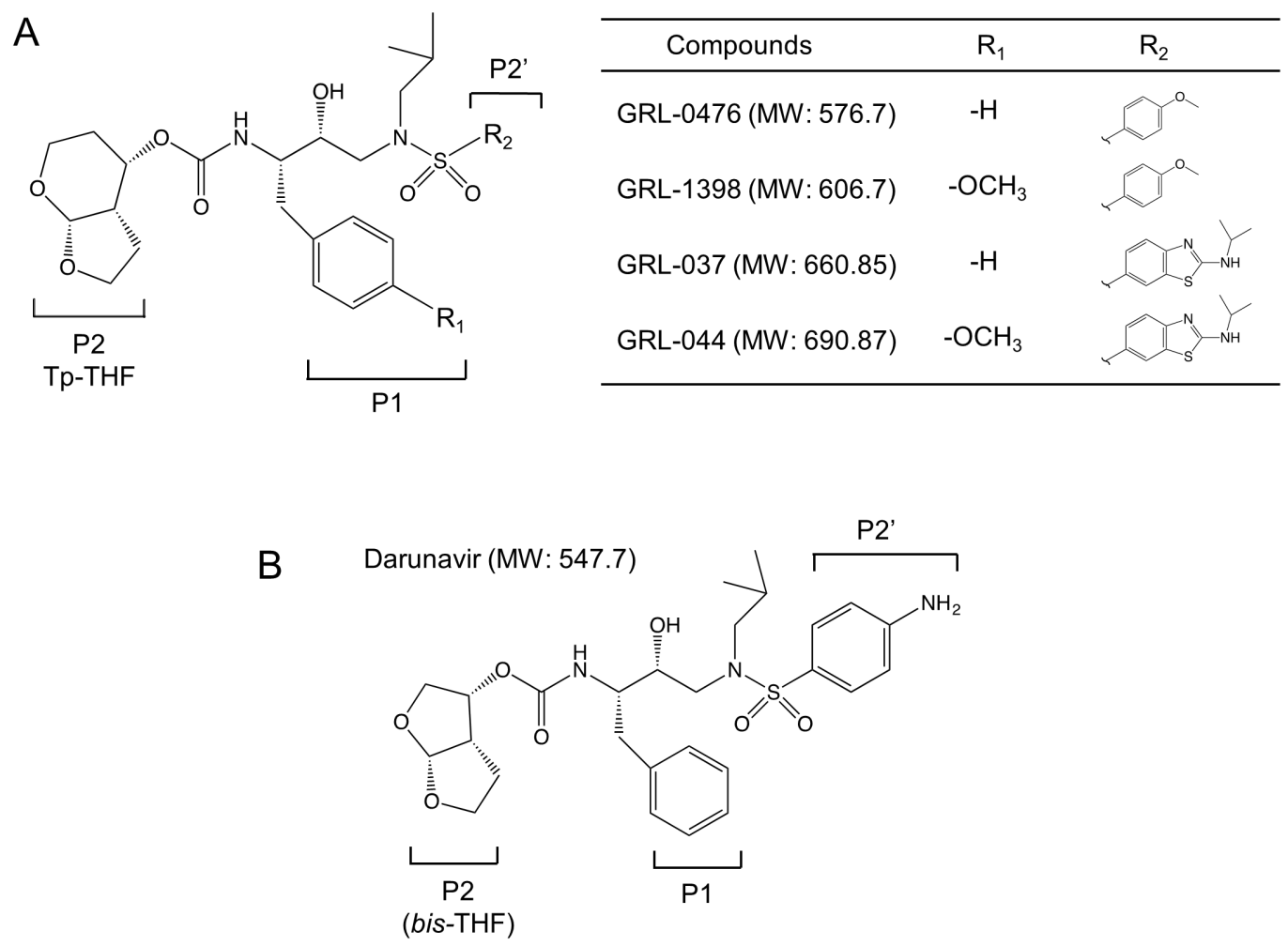

Figure 1. Structures of GRL-0476, GRL-1398, GRL-037, and GRL-044. Structures of GRL-0476, GRL-1398, GRL-037, and GRL-044 (Panel A). The structure of darunavir is shown as a reference (Panel B). 
Table 2. Antiviral activity of GRL-044 against HIV-1 ${ }_{\mathrm{NL4}-3}$ and $\mathrm{HIV}-2_{\mathrm{EHO}}$ and its cytotoxicity in vitro

\begin{tabular}{|c|c|c|c|c|}
\hline \multirow{2}{*}{ Drug } & \multicolumn{2}{|c|}{ Mean $\mathrm{IC}_{50}(\mathrm{nM}) \pm \mathrm{SD}$} & \multirow{2}{*}{$\mathrm{CC}_{50}(\mu \mathrm{M})$} & \multirow{2}{*}{ Selectivity index ${ }^{a}$} \\
\hline & $\mathrm{HIV}-1_{\mathrm{NL4}-3}$ & HIV-2 ${ }_{\text {ЕHO }}$ & & \\
\hline APV & $39 \pm 12$ & $375 \pm 63$ & $152 \pm 6$ & 3,900 \\
\hline ATV & $2.6 \pm 0.3$ & $60 \pm 23$ & $40 \pm 1$ & 15,400 \\
\hline DRV & $3.3 \pm 0.4$ & $3.8 \pm 1.2$ & $145 \pm 12$ & 43,900 \\
\hline GRL-044 & $0.0028 \pm 0.0008$ & $0.0004 \pm 0.0002$ & $52 \pm 3$ & $18,571,000$ \\
\hline
\end{tabular}

The data shown represent mean values ( \pm 1 standard deviation) derived from the results of three independent experiments. ${ }^{\mathrm{a}}$ Each selectivity index denotes a ratio of $\mathrm{CC}_{50}$ in MT- 4 cells to $\mathrm{IC}_{50}$ against HIV-1 $1_{\mathrm{NL} 4-3}$.

comparably potent $\left(\mathrm{IC}_{50}=3.3\right.$ and $3.5 \mathrm{nM}$, respectively $)$ even as compared to the least active experimental PI, GRL-0476, tested in the present study (Table 1).

GRL-044 exerts potent activity against PI-resistant and multi-drug-resistant $H I V-1$ variants

We next examined whether GRL-044 was active against a variety of HIV-1 variants that had been selected in vitro with each of three FDA-approved PIs: APV, ATV, and LPV (Table 1). Those HIV-1 variants had been selected in vitro in the presence of increasing concentrations of each PI $(16,17)$. All the three variants were confirmed to have multiple amino acid substitutions in the virally-encoded protease, which have reportedly been associated with viral resistance to PIs (see the footnotes of Table 1). Two in vitro DRVselected HIV-1 variants, $\mathrm{HIV}_{\mathrm{DRV}}{ }^{\mathrm{R}_{10}}$ and $\mathrm{HIV}_{\mathrm{DRV}} \mathrm{R}_{\mathrm{P} 30}$, were obtained by propagating a mixture of eight multidrug resistant HIV-1 variants $\left(\mathrm{HIV}_{\text {BMIX }}\right)$ in the presence of increasing concentrations of DRV (17). Each of the PI-selected variants ( $\mathrm{HIV}_{\mathrm{APV}-5 \mu \mathrm{M}}, \mathrm{HIV}_{\mathrm{ATV}-5 \mathrm{MM}}$, and $\mathrm{HIV}_{\mathrm{LPV}-}$ ${ }_{5 \mu M}, H_{I V}{ }_{D R V}{ }^{{ }_{P} 10}, H_{I V} V_{D R V}{ }^{R_{P 30}}$ ) was highly resistant to the corresponding PI, which the variant was selected against, and the fold differences in the $\mathrm{IC}_{50} \mathrm{~s}$ relative to the $\mathrm{IC}_{50}$ of each drug against $\mathrm{HIV}-1_{\mathrm{NL} 4-3}$ ranged from $>14$ to $>303$, although ATV exerted potent activity against $\mathrm{HIV}_{\mathrm{APV}-5 \mu \mathrm{M}}$ (Table 1). Two multi-drug-resistant HIV-1 strains, $\mathrm{HIV}_{\mathrm{B}}$ and $\mathrm{HIV}_{\mathrm{C}}$, that were isolated from patients experiencing treatment failure following longterm ART (14), were also employed. The three FDAapproved PIs were all least active against $\operatorname{HIV}_{B}$ and $\mathrm{HIV}_{\mathrm{C}}$. DRV was moderately active against $\mathrm{HIV}_{\mathrm{ATV}-5 \mu \mathrm{M}}$, $\mathrm{HIV}_{\mathrm{B}}$, and $\mathrm{HIV}_{\mathrm{C}}$, while it had lost its activity against $\mathrm{HIV}_{\text {APV } 5 \mu \mathrm{M}}, \mathrm{HIV}_{\mathrm{LPV}-5 \mu \mathrm{M}}, \mathrm{HIV}_{\mathrm{DRV}} \mathrm{R}_{\mathrm{P} 10}$, and $\mathrm{HIV}_{\mathrm{DRV}} \mathrm{R}_{\mathrm{P} 30}$. GRL-0476 and GRL-1398 were substantially less active against $\mathrm{HIV}_{\mathrm{DRV}} \mathrm{R}_{\mathrm{P} 10}$ and $\mathrm{HIV}_{\mathrm{DRV}} \mathrm{R}_{\mathrm{P} 30}$ with the foldchanges ranging from 80 to 598 . GRL-037 also lost its activity to most of the variants examined with $\mathrm{IC}_{50}$ values ranging from 5.5 to $83 \mathrm{nM}$, with fold-changes ranging from 131 to 1,976 , while it retained its activity against $\mathrm{HIV}_{\mathrm{APV}-5 \mu \mathrm{M}}$ with an $\mathrm{IC}_{50}$ value of $0.44 \mathrm{nM} 10$ fold change. GRL-044, the most potent PI against HIV- $1_{\text {NL4-3 }}$ among the two novel PIs, also decreased in potency against all the HIV variants examined with fold-changes ranging from 20 to 5,758 . However, its
$\mathrm{IC}_{50}$ values remained substantially low, with a range from 0.065 to $19 \mathrm{nM}$ (Table 1).

GRL-044 is potent against HIV-2 $2_{E H O}$ with favorable toxicity profiles

In general, PIs that are active against PI-resistant HIV1 variants are also active against HIV-2 strains since most amino acid residues substituted for HIV-1 to develop resistance against PIs, reside in wild-type HIV2 strains $(19,21)$. We thus examined GRL-044 together with APV, ATV, and DRV against HIV-2 $2_{\text {Ено }}$ in the drug susceptibility assays. As expected, both APV and ATV, which significantly lose their activity against various PI-resistant HIV-1 variants (Table 1), had significantly greater $\mathrm{IC}_{50}$ values against HIV-2 $2_{\mathrm{EHO}}$ (Table 2). DRV, which exerts its potent activity against various HIV1 variants resistant to other FDA-approved PIs [except for saquinavir $(\mathrm{SQV})(8)$, had a comparable potency against HIV- $2_{\mathrm{EHO}}$ compared to that against HIV- $1_{\mathrm{NL} 4-3}$. Since GRL-044 was highly potent against various highly drug-resistant HIV-1 variants (except against $\mathrm{HIV}_{\mathrm{DRV}} \mathrm{R}_{\mathrm{P} 30}$ ) with $\mathrm{IC}_{50}$ values ranging from 0.065 to 4.6 $\mathrm{nM}$ (Table 1), we also examined whether GRL-044 was active against $\mathrm{HIV}-2_{\mathrm{Eно}}$ as well. Interestingly, GRL044 proved to be significantly more potent (by 7 -fold) against $\mathrm{HIV}-2_{\mathrm{EHO}}\left(\mathrm{IC}_{50}=0.0004 \mathrm{nM}\right)$ than against HIV$1_{\mathrm{NL4}-3}\left(\mathrm{IC}_{50}=0.0028 \mathrm{nM}\right)$ (Table 2). Such a feature was seen in GRL-142, which is more potent against most of PI-resistant HIV-1 variants examined than against wild-type HIV-1 strain (19). It is of note that GRL-044 showed a highly favorable selectivity index of 18,571,000 compared to those of the three FDAapproved PIs, APV, ATV, and DRV (Table 2).

Greater thermal stability of $P R^{W T}$ in the presence of GRL-044 compared to that of SQV or DRV

In order to examine the specificity of possible binding of GRL-037 and GRL-044 to protease, thermal stability of $\mathrm{PR}^{\mathrm{WT}}$ in the absence or presence of AZT, SQV, DRV, GRL-037 or GRL-044 was determined using the differential scanning fluorimetry employing SYPRO $^{\circledR}$ orange as previously described $(18,19)$. The $T_{m}$ (50\% melting temperature) values were determined as the temperature, at which the relative fluorescent 
A

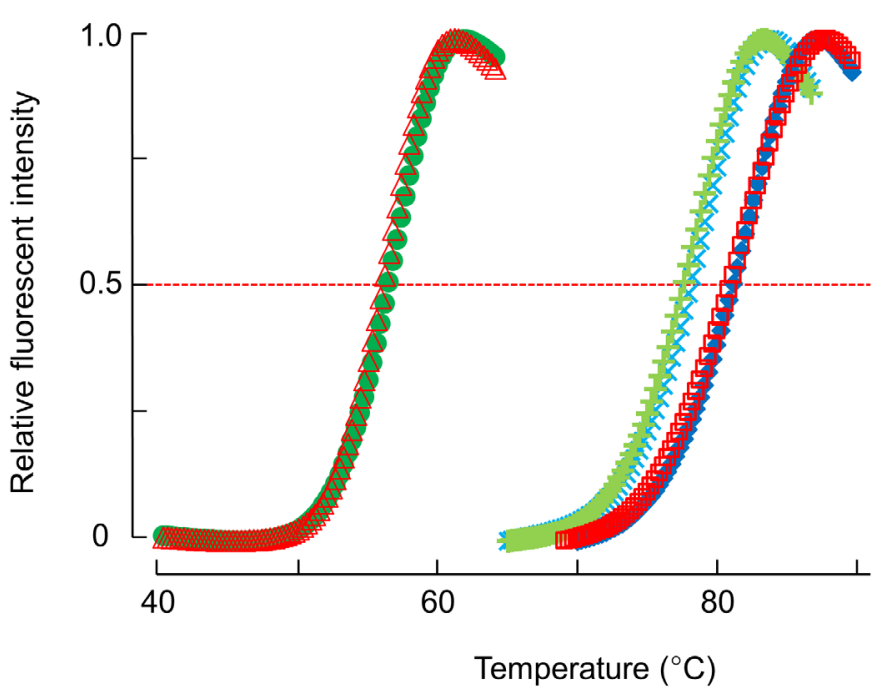

B

\begin{tabular}{ccc}
\hline \multicolumn{2}{c}{ Compound } & Mean $\operatorname{Tm}\left({ }^{\circ} \mathrm{C}\right) \pm \mathrm{SD}(\Delta \mathrm{Tm})$ \\
\hline & No drug & $56.3 \pm 0.2$ \\
$\Delta \quad$ AZT & $56.0 \pm 0.2(-0.3)$ \\
$+\quad$ SQV & $77.5 \pm 0.1(21.2)$ \\
$\times \quad$ DRV & $78.0 \pm 0.2(21.7)$ \\
$\bullet$ GRL-037 & $81.0 \pm 0.1(24.7)$ \\
$\square$ GRL-044 & $81.0 \pm 0.2(24.7)$
\end{tabular}

Figure 2. Thermal stability of $P^{\mathrm{wT}}$ with AZT, DRV, SQV, GRL-037, or GRL-044 as determined using differential scanning fluorimetry (DSF). Relative fluorescence intensities determined by DSF using SYPRO orange (Panel A). $T_{m}$ values shown represent the temperatures at which the relative fluorescence intensity was 0.5 , and $\Delta T_{m}$ values indicate the Tm difference between protease complexed with each compound and that with no compound (Panel B).

intensity became 50\%. As shown in Figure 2, the mean $T_{m}$ values without agent and with a nucleoside reverse transcriptase inhibitor (NRTI), zidovudine (AZT), were $56.3^{\circ} \mathrm{C}$ and $56.0^{\circ} \mathrm{C}$, respectively, while those with DRV and SQV were comparably greater with $77.5^{\circ} \mathrm{C}$ and $78.0^{\circ} \mathrm{C}$, respectively. The mean $T_{m}$ values of GRL-037 and the most potent GRL-044 were also comparably high with $81.0^{\circ} \mathrm{C}$. It is of note that the antiviral potency of GRL-044 against HIV-1 $1_{\text {NL4-3 }}$ was greater than that of GRL-037 by approximately 12-folds, although the $T_{m}$ values of both compounds were comparable.

In vitro selection of $H I V-1$ variants resistant to $G R L$ 044 .

In an attempt to relatively quickly and effectively select HIV-1 variants in vitro, we previously used a mixture of 8 and 11 multi-PI-resistant clinical HIV-1 isolates $\left(\mathrm{HIV}_{\mathrm{MDRs}}, \mathrm{HIV}_{8 \mathrm{MIX}}\right.$ and $\mathrm{HIV}_{11 \mathrm{MIX}}$, respectively, as a starting HIV-1 population, resulting in successful rapid emergence of highly DRV- or TPV-resistant HIV-1 variants $(15,17)$. We, therefore, employed HIV $\mathrm{H}_{1 \mathrm{MIX}}$ to possibly successfully obtain resistant HIV-1 variants against GRL-044 in the present study (Figure 3). Figure 4 illustrates the amino acid sequences of protease of each of the $11 \mathrm{HIV}_{\text {MDRs. }}$. When selected in the presence of increasing concentrations of APV, the virus quickly became resistant to the drug and was capable of replicating even in the presence of a high concentration, $5 \mu \mathrm{M}$, by 13 and 16 weeks of selection in the two independently conducted selection experiments (Figure 3 ). The HIV $\mathrm{H}_{11 \mathrm{MIX}}$ also acquired resistance against DRV as well and became capable of replicating in the presence of $5 \mu \mathrm{M}$ DRV, by 29 weeks of selection (Figure 3, Experiment 1). However, as depicted in the left panel of

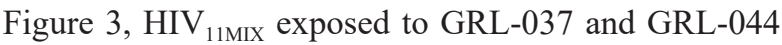
did not replicate in the presence of $2 \mu \mathrm{M}$ even at week 40 of the passage. In an attempt to reproduce the selection feature of Experiment 1, we conducted the second selection assay under similar conditions. As shown in the right panel of Figure 3, the selection feature was virtually the same and HIV $\mathrm{H}_{1 \mathrm{MIX}}$ failed to replicate in the presence of relatively high concentrations of GRL-044. No further attempt to select GRL-044-resistant variants was made and the selection was terminated at week 27.

The homologous recombination-based selection expedites the emergence of resistance-associated amino acid substitutions

We determined the amino acid sequences by deducing from nucleic acid sequences of the protease of $\mathrm{HIV}_{\text {11MIX }}$ exposed to APV, DRV, GRL-037, and GRL-044 over $13,29,40$, and 40 weeks in experiment $1\left(\mathrm{HIV}_{11 \mathrm{MIX}}{ }^{\mathrm{APV}-}\right.$ 1-WK13, HIV ${ }_{11 \mathrm{MIX}}{ }^{\text {DRV-1-WK29, }} \mathrm{HIV}_{11 \mathrm{MIX}}{ }^{\text {037-1-WK40, }}$, and $\mathrm{HIV}_{11 \mathrm{MIX}}{ }^{044-1-\mathrm{WK} 40}$ ) respectively (Figure 5). The amino acid sequences showed distinctive differences between the former 2 strains and the latter 2 strains. The amino acid sequences of $\mathrm{HIV}_{11 \mathrm{MIX}}{ }^{\mathrm{APV}-1-\mathrm{WK} 13}$ and $\mathrm{HIV}_{11 \mathrm{MIX}}{ }^{\text {DRV-1- }}$ wK29 revealed that both selected variants had acquired V32I substitution as shown in green in Figure 5, which none of the eleven multi-PI-resistant clinical HIV1 variants had contained before the selection as far as judged from the direct sequencing conducted for each of HIV $_{11 \mathrm{MIX}}$ (Figure 4). One reason why these two variants have similarity in the amino acid substitution patterns 


\section{A. Experiment 1}

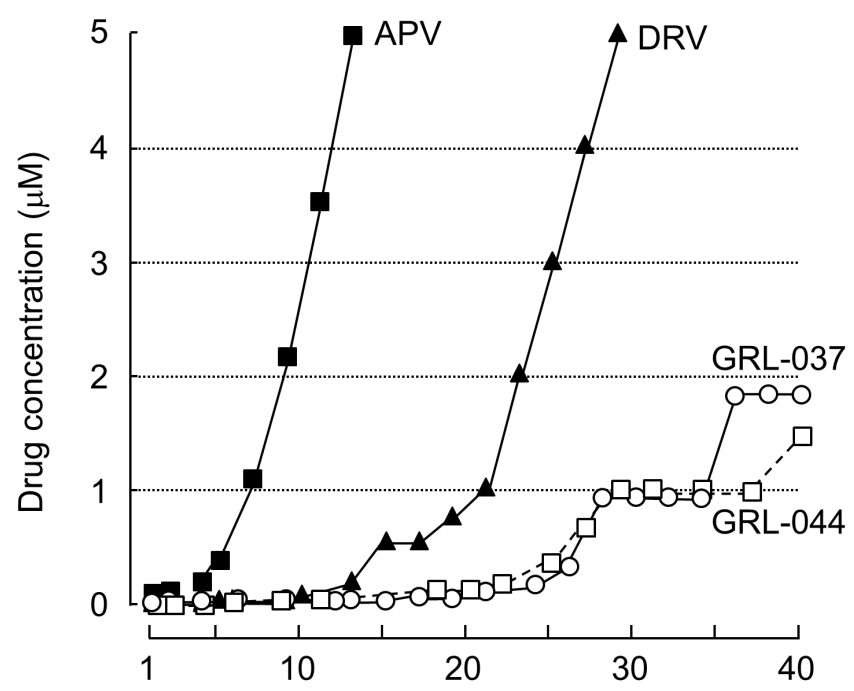

\section{B. Experiment 2}

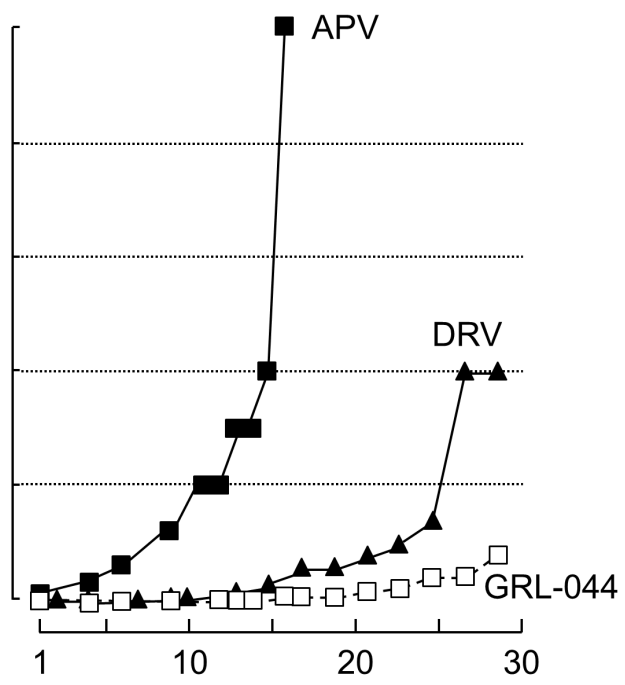

Weeks

Figure 3. In vitro selection of HIV-1 variants against GRL-037, GRL-044, APV, and DRV. A mixture of 11 multi-PI-resistant HIV-1 isolates (HIV 11MIX ) was propagated in MT-4 cells in the presence of increasing concentrations of GRL-037, GRL-044, APV, or DRV. The selection was conducted by passage of cell-free virus. Amino acid substitutions appeared in each HIV-1 during selection are illustrated in Figure 5.

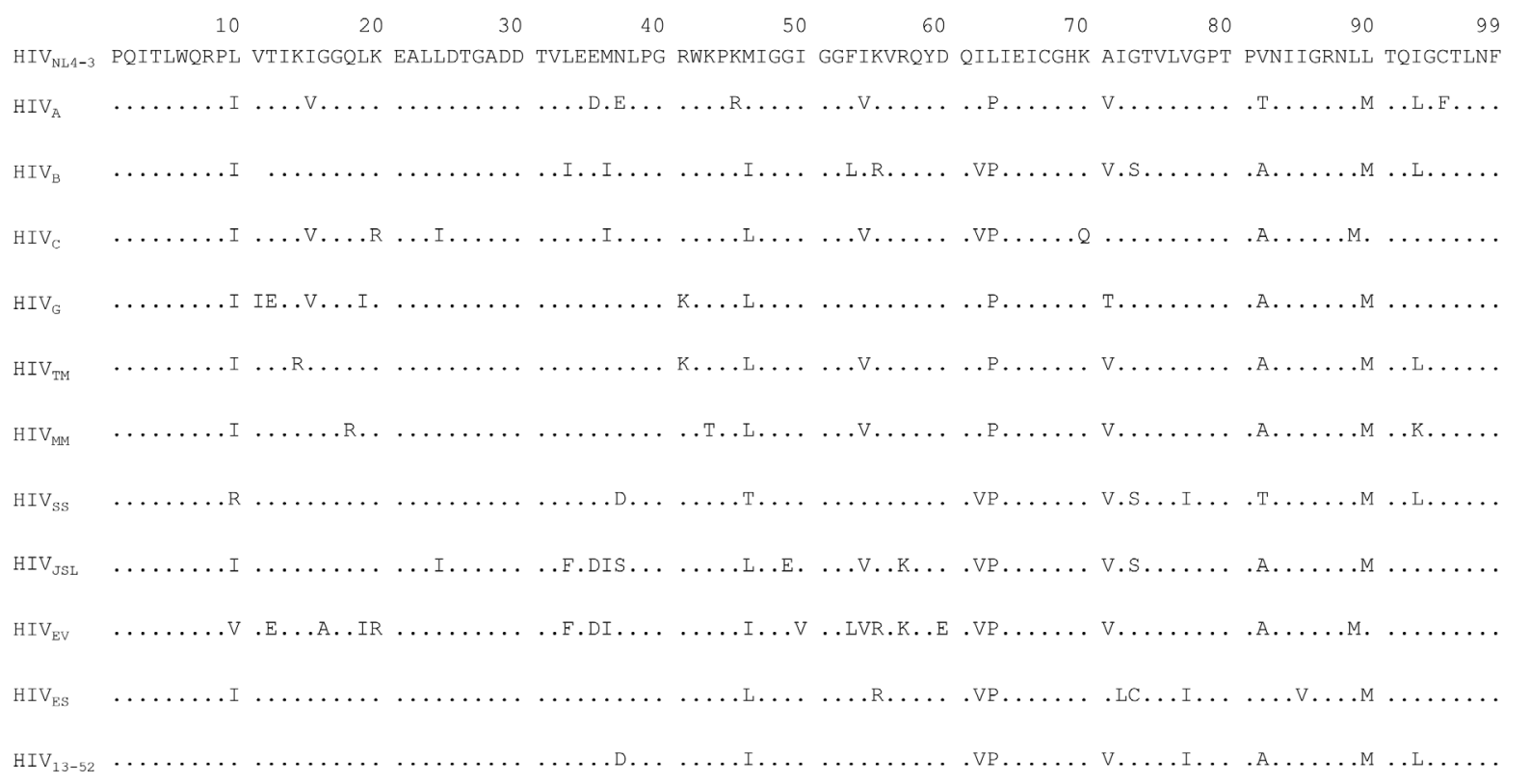

Figure 4. Amino acid sequence of protease of 11 multi-PI-resistant HIV-1 isolates. The amino acid sequences of protease deduced from nucleotide sequences of the protease-encoding region of each of the eleven multi-PI-resistant HIV-1 isolates are shown. The consensus sequence of $\mathrm{HIV}_{\mathrm{NL4-3}}$ is illustrated at the top as a reference. Identity with sequence at individual amino acid positions is indicated by dots.

in protease, i.e., the acquisition of V32I, is that APV and DRV share structural similarity $(8)$. HIV $_{11 \mathrm{MIX}}{ }^{\mathrm{DRV}-1-}$ wK29 had also acquired I84V substitution (highlighted in blue in Figure 5), which often emerges when HIV1 becomes highly resistant to various PIs $(17,22)$, often resulting in conferring significant resistance to PIs on
HIV-1 (22,23). It is thought that the emergence of V32I and $\mathrm{I} 84 \mathrm{~V}$ took place relatively quickly because of the

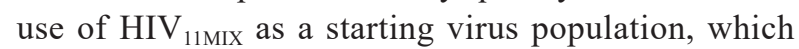
led to homologous recombination occurring from one isolate to another in the presence of escalating doses of DRV, expediting the emergence of highly DRV-resistant 


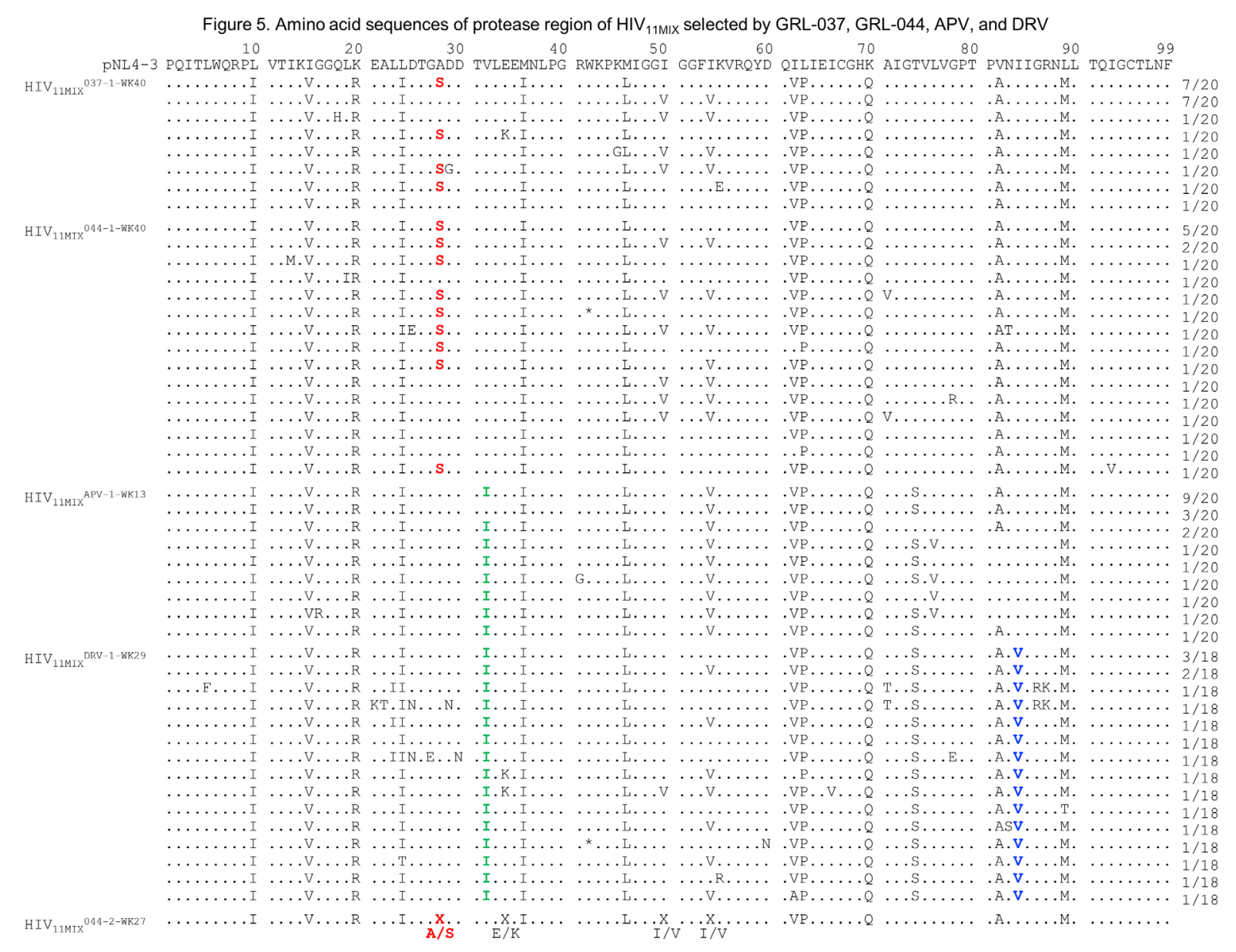

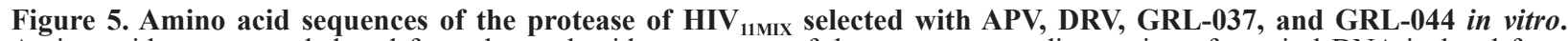
Amino acid sequences deduced from the nucleotide sequences of the protease-encoding region of proviral DNA isolated from HIV $_{11 \mathrm{MIX}}$ selected with APV at 13 weeks, DRV at 29 weeks, GRL-037 at 40 weeks, and GRL-044 at 40 weeks in experiment 1 and GRL-044 at 27 weeks in experiment 2 are shown. The consensus sequence of HIV $\mathrm{NL4}_{-3}$ is illustrated at the top as a reference. Identity with sequence at individual amino acid positions is indicated by dots. Fractions on the right are the number of viruses which each clone is presumed to have originated from over the total number of clones examined. The amino acid sequence of $\mathrm{HIV}_{11 \mathrm{MIX}}{ }^{04-2-\mathrm{WK} 27}$ was determined by the deduction of the direct nucleic acid sequence obtained for $\mathrm{HIV}_{11 \mathrm{MIX}}{ }^{04-2-\mathrm{WK}_{2} 7}$.

HIV-1 variants (22).

Interestingly, in both $\mathrm{HIV}_{11 \mathrm{MIX}}{ }^{037-1-\mathrm{WK} 40}$, and HIV $_{11 \mathrm{MIX}}{ }^{04-1-\mathrm{WK} 40}$, the unique substitution, A28S, known to lead to a significant reduction in protease activity (24) and poor viral fitness (25), had been acquired by passage 40 (highlighted in red in Figure 5), while both resultant variants had not apparently acquired high levels of resistance to GRL-037 or GRL-044 and did not replicate well in the presence of either of the two compounds (Figure 3). Since the A28S substitution has been seen with a few PIs, such as TMC-126 (26), GRL-98065 (27), brecanavir (25), and GRL-1398 (12) and not with any of the currently available FDA-approved PIs except only in a few cases $(28,29)$, we repeated the selection assay with APV, DRV, and GRL-044 (Figure 3 , Experiment 2). When we determined the amino acid sequence of the protease of $\mathrm{HIV}_{11 \mathrm{MIX}}$ with direct nucleic acid sequencing after HIV $_{11 \text { IIX }}$ was selected in the presence of GRL-044 in the second selection assay, the same A28S substitution was identified by week 27 as highlighted in red at the bottom of Figure 5 .
Structural interactions of GRL-044 and DRV with wildtype protease.

We finally attempted to determine the structural interactions of GRL-044 with wild-type protease $\left(\mathrm{PR}^{\mathrm{WT}}\right)$. In this attempt, we started from the crystal structure of GRL-142 complexed with $\mathrm{PR}^{\mathrm{WT}}$ and modified the structure of GRL-142 (19) to generate GRL-044-PR ${ }^{\mathrm{WT}}$. A full minimization of GRL-044-PR ${ }^{\mathrm{WT}}$ using OPLS3 force field was carried out to interrogate the structural interactions. We also wished to compare the interactions with those of DRV with $\mathrm{PR}^{\mathrm{WT}}$. Starting from a crystal structure of DRV-PR ${ }^{\mathrm{WT}}$ (20), hydrogen atoms were added, followed by assignment of protonation states of aspartates and a full minimization using OPLS3 force field. The hydrogen bond and van der Waals surface interactions in GRL-044-PR ${ }^{\mathrm{WT}}$ and DRV-PR ${ }^{\mathrm{WT}}$ were subsequently analyzed and compared. The analyses helped understand the similarities and differences of polar and non-polar interactions of these two inhibitors complexed with $\mathrm{PR}^{\mathrm{WT}}$. 

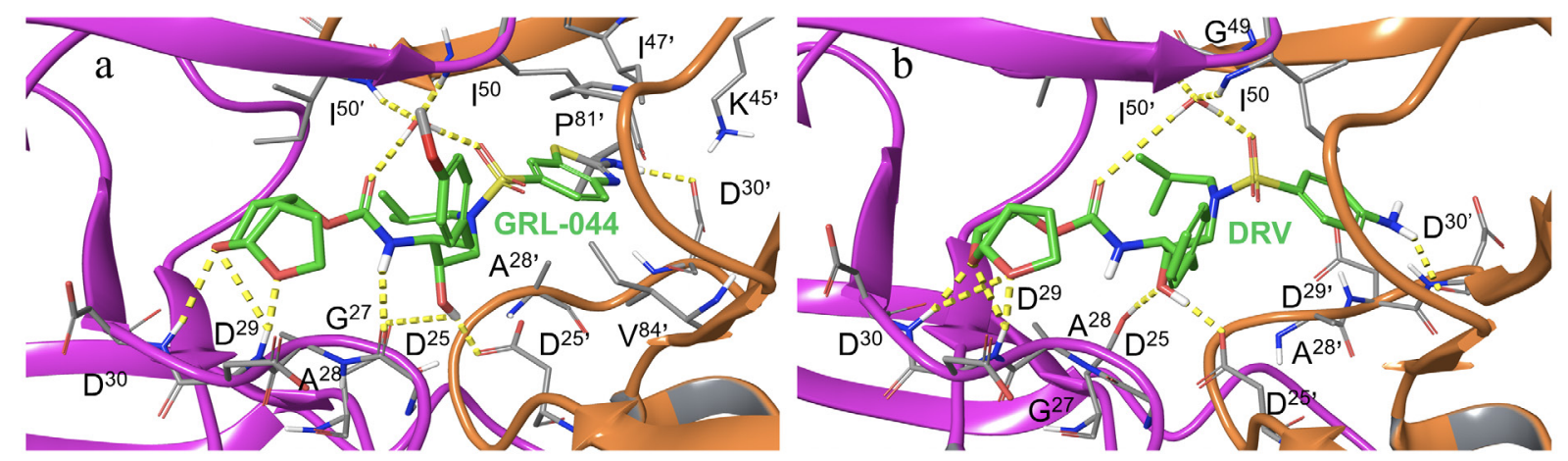

Figure 6. Polar interactions of GRL-044 and DRV with wild-type protease. The polar interactions of GRL-044 and DRV with wild-type protease are shown in Panels-a and -b, respectively. The protease dimer is shown in a ribbon representation, with the monomers shown in purple and orange. The residues in the immediate vicinity of the inhibitors $(<3 \AA)$ are shown. The hydrogen bond interactions are shown by yellow dotted lines. GRL-044 and DRV are in green carbons, and thick sticks, the protease residues are in gray carbons.

The polar interactions of GRL-044 with $\mathrm{PR}^{\mathrm{WT}}$ are shown in Figure 6a. The Tp-THF moiety of GRL-044 has hydrogen bond interactions with D29 and D30. The carbonyl and sulfonyl oxygens form polar interactions with I50 and I50' present in the protease flap through a bridging water molecule. This interaction with the protease flap is seen for various PIs $(8,14,19)$. An amine group of the inhibitor makes a hydrogen bond interaction with G27 and the hydroxyl group of the inhibitor makes hydrogen bond interactions with the catalytic aspartates. The amine from the benzothiazole in the P2' group makes a hydrogen bond interaction with the side chain of D30'. DRV has many of these polar interactions with $\mathrm{PR}^{\mathrm{WT}}$. The residues identified within $3 \AA$ distance from the inhibitors are shown in panels a and $b$. Because of the slightly larger size of GRL-044 over DRV, there are a few additional residues of $\mathrm{PR}^{\mathrm{WT}}$ that are closer to the former.

We then focused on the moieties that are different between GRL-044 and DRV and examined how the identified differences impact the van der Waals interactions with $\mathrm{PR}^{\mathrm{WT}}$. GRL-044 has an Ip-Abt as P2' ligand compared to the aminobenzene of DRV. The P2'Ip-Abt has a better van der Waals interaction with $\mathrm{D}^{2} 0^{\prime} /$ K45' than the aminobenzene of DRV (Figure 7a, b). The P2' moiety of GRL-044 is also closer to R8, forming weak van der Waals interactions, whereas the R8 group is much further away from the aminobenzene of DRV (Figure 7c, d). While both P2-Tp-THF of GRL-044 and P2-bis-THF of DRV have van der Waals surface contact with I47, the P2'-Ip-Abt of GRL-044 has a better van der Waals contact with I47' than does the P2'-aminobenzene of DRV (Figure 7e, f). GRL-044 also makes better contact with G48 than does DRV (Figure 7g, h). The P1-paramethoxyphenyl substituent of GRL-044 makes better contact with G49 than the phenyl substituent alone of DRV (Figure 7i, j). Of note, residues 47-49 are in the flap region of the protease. Interactions of an inhibitor with the flap region probably help keep the region in a closed conformation resulting in stronger binding $(11,30)$.

A28 is seen present in the active site cavity. The P2bis-THF of DRV and P2-Tp-THF of GRL-044 have van der Waals contacts with A28. Interestingly, both P2'-IpAbt of GRL-044 and P2'-aminobenzene of DRV also have van der Waals contacts with A28'. The van der Waals surface contact of P2'-Ip-Abt of GRL-044 with A28' appears to be better compared to aminobenzene of DRV because of the larger surface of Ip-Abt (Figure $7 \mathrm{k}, 1)$. Whether these interactions are wholly or partially responsible for the emergence of A28S substitution is not known at this time and needs to be explored.

Overall, the improved van der Waals interactions of GRL-044 with multiple PR ${ }^{\mathrm{WT}}$ residues located in different protease domain should explain at least in part why GRL-044 has the significantly stronger binding to $\mathrm{PR}^{\mathrm{WT}}$ and greater antiviral potency over DRV (Table 1).

\section{Discussion}

In the present work, we designed, synthesized, and identified two novel nonpeptidic PIs, GRL-037 and GRL-044, containing (i) P2-Tp-THF, (ii) P1-benzene and P1-methoxybenzene, respectively, and (iii) P2'-IpAbt (Figure 1). GRL-044 that contains (i) P2-Tp-THF, (ii) P1- methoxybenzene, and (iii) P2'-Ip-Abt proved to be most potent against $\mathrm{HIV}-1_{\mathrm{NL} 4-3}$ with $\mathrm{IC}_{50}$ values of $0.0028-0.0033 \mathrm{nM}$ (Tables 1 and 2). The $\mathrm{IC}_{50}$ values against various PI-resistant HIV-1 variants also proved to be highly favorable ranging from 0.065 to $19 \mathrm{nM}$ (Table 1). We have previously described that GRL-077 and GRL-058, both of which contain P2-C5-modified Tp-THF, P1-methoxybenzene, and P2'-Ip-Abt were potent against $\mathrm{HIV}-1_{\mathrm{NL4}-3}$ and various PI-resistant HIV1 variants (30). But the $\mathrm{IC}_{50}$ value of GRL-077 and GRL-058 were 21 and $3.5 \mathrm{nM}$, less potent by $6,364-$ and 1,061-fold, respectively than GRL-044. There is only one difference: both GRL-077 and GRL-058 have C5-modified Tp-THF, while GRL-044 has unmodified 

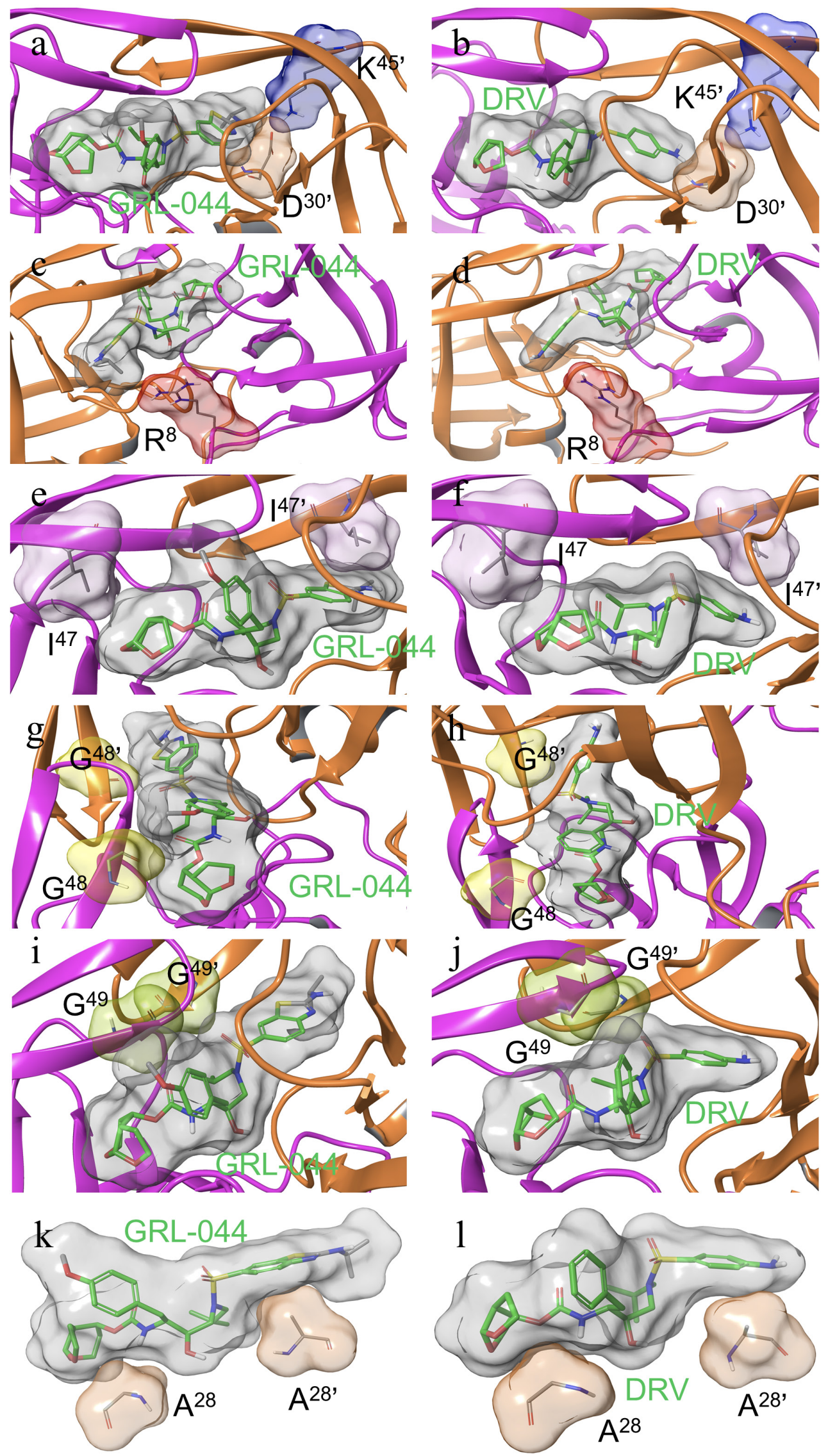

Figure 7. van der Waals surface interactions of GRL-044 and DRV with wild-type protease. The van der Waals surface interactions of GRL-044 and DRV with important residues are shown in panels a-l. The inhibitor surfaces are shown in gray, D30' is in orange, K45' in blue, R8 in red, I47 and I47' in plum, G48 and G48' in yellow, G49 and G49' in yellowish green, A28 and A28' in orange. 
Tp-THF, strongly suggesting that the C5-modification significantly weakens the potency against HIV $-1_{\mathrm{NL} 4-3}$, although the potency of GRL-077 and GRL-058 against various PI-resistant variants including $\mathrm{HIV}_{\mathrm{DRV}}{ }^{\mathrm{R} 10}$ and $\mathrm{HIV}_{\mathrm{DRV}} \mathrm{R}_{\mathrm{P} 30}$ is comparable with that of GRL-044.

Determination of thermal stability using the differential scanning fluorimetry employing SYPRO ${ }^{\circledR}$ orange helps understand the specificity and binding force of protein and its ligand $(19,29,31)$. The $T_{m}$ values of DRV and SQV, which were greater than those without agent and with AZT, were the lowest and those with GRL-037 and GRL-044 were further greater (Figure 3). However, in the present study, the mean $T_{m}$ values of GRL-037 and the most potent GRL-044 were virtually identical, while GRL-044 was more potent by $\sim 12$-folds than GRL-037, showing that the $T_{m}$ values in the DSF assay are not always proportionate to the biological activity of compounds such as antiretroviral potency.

It has been demonstrated that upon reverse transcription, reverse transcriptase (RT) frequently switches a template from one viral genomic strand to another, producing recombinant proviral DNA or a mosaic proviral DNA containing multiple parent genomic pieces. In fact, a single $\mathrm{CD} 4^{+}$target cell can be infected with multiple HIV-1 virions $(32,33)$. If newly produced proviral DNA has mutations in one spot conferring HIV-1 resistance to one drug and mutations in the other spot that are associated with resistance to the other drug, the daughter virions acquire resistance to both drugs, a process called homologous recombination (34-36). Thus, homologous recombination is likely to accelerate the development of multi-drug and multiclass drug resistance in the setting of HIV-1 infection. As we previously demonstrated, when the selection of HIV-1 variants with DRV was attempted using a single viral strain as the starting virus population, no DRVresistant variants emerged; however, when a mixture of eight multi-drug-resistant (as well as multi-PI-resistant) clinical HIV-1 strains $\left(\mathrm{HIV}_{8 \mathrm{MIX}}\right)$ was employed as the starting virus population, highly DRV-resistant HIV1 variants such as $\operatorname{HIV}_{\mathrm{DRV}}{ }^{\mathrm{R} 10}$ and $\mathrm{HIV}_{\mathrm{DRV}}{ }_{\mathrm{P} 30}$ were relatively quickly obtained, highly likely through the homologous recombination $(17,23)$. In the current homologous recombination-based selection assay, HIV $_{11 \mathrm{MIX}}$ exposed to APV and DRV over 13 and 29 weeks, resulting in $\mathrm{HIV}_{11 \mathrm{MIX}}{ }^{\mathrm{APV}-1-\mathrm{WK} 13}$ and $\mathrm{HIV}_{11 \mathrm{MIX}}{ }^{\mathrm{DRV}-}$ 1-WK29, respectively, had acquired V32I substitution (highlighted in green in Figure 5). The V32I substitution does not appear alone when wild-type HIV-1 $\left(\mathrm{HIV}^{\mathrm{WT}}\right)$ as a starting virus population is selected with DRV, since V32I confers on HIV $^{\text {WT }}$ greater susceptibility ( $\sim$ 14-fold) to DRV as well as a highly compromised replication fitness (22). The V32I substitution is, therefore, thought to be a key substitution, which hardly emerges when selected in test tube and in the clinical setting; but once V32I substitution emerges with other amino acid substitutions, the V32I substitution predisposes HIV-
1 to quickly develop high-level DRV-resistance. It is thought that the emergence of V32I took place relatively quickly because of the use of $\mathrm{HIV}_{11 \mathrm{MIX}}$ as a starting virus population, which led to the homologous recombination occurring from one isolate to another in the presence of escalating doses of DRV, expediting the emergence of highly DRV-resistant HIV-1 variants (22).

It is noteworthy that, when $\mathrm{HIV}_{11 \mathrm{MIX}}$ was selected with GRL-037 or GRL-044, the A28S substitution, known to lead to a $\sim 1,500$-fold reduction in $\mathrm{kcat} / \mathrm{Km}$ in protease activity (24) and the poor viral fitness (25), had been acquired by passage 40 (Figure 5), although both resultant variants $\mathrm{HIV}_{11 \mathrm{MIX}}{ }^{037-1-\mathrm{WK} 40}$ and $\mathrm{HIV}_{11 \mathrm{MIX}}{ }^{044-1-}$ wK40 were not much resistant to either of the compounds (Figure 3 ). The reason why both HIV $_{11 \mathrm{MIX}}{ }^{037-1-\mathrm{WK} 40}$ and $\mathrm{HIV}_{11 \mathrm{MIX}}{ }^{044-1-\mathrm{WK} 40}$ were not sufficiently replicative in the homologous recombination-based selection assay could be due to the acquisition of A28S substitution. The A28S substitution has been seen in the selection assay of HIV-1 with a few PIs, such as TMC-126 (26), GRL-98065 (27), brecanavir (25), and GRL-1398 (12), and not with any of the currently available FDA-approved PIs except only in a few cases $(28,29)$. Of note, however, the population size of HIV-1 in the present homologous recombinationbased selection assay is assumed to be significantly smaller than the size of HIV-1 variants such as polymorphic amino acid substitution-containing variants that reside in HIV-1-infected individuals, the appearance of mutations is affected by stochastic phenomena. Therefore, the rates of mutation appearance in culture may not be reliable. Moreover, in the initial selection, we observed the appearance of the rare A28S substitution in both $\mathrm{HIV}_{11 \mathrm{MIX}}{ }^{037-1-\mathrm{WK} 40}$ and $\mathrm{HIV}_{11 \mathrm{MIX}}{ }^{044-1-\mathrm{WK} 40}$. Thus, we repeated the selection assay with GRL-044. As shown in the bottom line of Figure 5, the A28S substitution was reproducibly identified through direct sequencing. Structurally, the P2-bis-THF of DRV and P2-Tp-THF of GRL-044 have van der Waals contacts with A28. Both P2'-Ip-Abt of GRL-044 and P2'-aminobenzene of DRV also have the contacts with A28'. However, apparently the van der Waals surface contact of P2'-Ip-Abt of GRL-044 with A28' appears to be better compared to aminobenzene of DRV because of the larger surface of Ip-Abt. Whether these interactions are wholly or partially responsible for the emergence of A28S substitution is not known at this time and needs to be explored.

Taken together, in our efforts to maximize the antiviral potency of the prototypic protease inhibitor, DRV (Figure 1), which has potent activity against various wild-type and drug-resistant HIV-1 species (8) and a reasonably high genetic barrier to the emergence of HIV-1 variants resistant to DRV $(17,18,37,38)$, we successfully designed and identified GRL-044, which has much greater potency and shows greater genetic barrier compared to the prototypic DRV. The structural mechanisms by which GRL-044 acquired the much greater potency than the potency of DRV appear to stem 
from the design of GRL-044 that the three moieties were substituted: (i) P2-Tp-THF, (ii) P1-methoxybenzene, and (iii) P2'-Ip-Abt, starting from the chemical structure of DRV. The present antiviral data and structural features of GRL-044 should shed lights in the further design and development of potent and "resistance-repellant" novel PIs.

\section{Acknowledgements}

The present work was supported in part by the Intramural Research Program of the Center for Cancer Research, National Cancer Institute, National Institutes of Health (HM); a grant from the National Institutes of Health (AI150466; AKG); a grant for Development of Novel Drugs for Treating HIV-1 Infection and AIDS from Japan Agency for Medical Research and Development (HM; JP15fk0410001 and JP18fk0410001); grants from Japan Society for the Promotion of Sciences; and a grant from National Center for Global Health and Medicine Research Institute. This study utilized the highperformance computational capabilities of the Biowulf Linux cluster at the National Institutes of Health, Bethesda, MD (http://hpc.nih.gov).

\section{References}

1. Cohen MS, Chen YQ, McCauley M, et al. Antiretroviral therapy for the prevention of HIV-1 transmission. N Engl J Med. 2016; 375:830-839.

2. Rodger AJ, Cambiano V, Bruun T, et al. Risk of HIV transmission through condomless sex in serodifferent gay couples with the HIV-positive partner taking suppressive antiretroviral therapy (PARTNER): final results of a multicentre, prospective, observational study. Lancet. 2019; 393:2428-2438

3. Feng M, Sachs NA, Xu M, Grobler J, Blair W, Hazuda DJ, Miller MD, Lai MT. Doravirine Suppresses Common Nonnucleoside Reverse Transcriptase InhibitorAssociated Mutants at Clinically Relevant Concentrations. Antimicrob Agents Chemother. 2016; 60:2241-2247.

4. Khalilieh S, Yee KL, Sanchez RI, Triantafyllou I, Fan L, Maklad N, Jordan H, Martell M, Iwamoto M. Results of a doravirine-atorvastatin drug-drug interaction study. Antimicrob Agents Chemother. 2017. 61. doi: 10.1128/ AAC.01364-16.

5. Yang LL, Li Q, Zhou LB, Chen SQ. Meta-analysis and systematic review of the efficacy and resistance for human immunodeficiency virus type 1 integrase strand transfer inhibitors. Int J Antimicrob Agents. 2019. doi: 10.1016/ j.ijantimicag.2019.08.008.

6. Landovitz RJ, Li S, Grinsztejn B, et al. Safety, tolerability, and pharmacokinetics of long-acting injectable cabotegravir in low-risk HIV-uninfected individuals: HPTN 077, a phase 2a randomized controlled trial. PLoS Med. 2018; 15:e1002690.

7. Ghosh AK, Leshchenko S, Noetzel M. Stereoselective photochemical 1,3-dioxolane addition to 5-alkoxymethyl$2(5 \mathrm{H})$-furanone: synthesis of bis-tetrahydrofuranyl ligand for HIV protease inhibitor UIC-94017 (TMC-114). J Org Chem. 2004; 69:7822-7829.
8. Koh Y, Nakata H, Maeda K, et al. Novel bistetrahydrofuranylurethane-containing nonpeptidic protease inhibitor (PI) UIC-94017 (TMC114) with potent activity against multi-PI-resistant human immunodeficiency virus in vitro. Antimicrob Agents Chemother. 2003; 47:31233129.

9. Potard V, Canestri A, Gallien S, Costagliola D, French Hospital Database on HIV. Use of darunavir in HIV-1infected individuals in routine clinical practice from 2012 to 2016 in France. J Antimicrob Chemother. 2019; doi: $10.1093 / \mathrm{jac} / \mathrm{dkz} 338$.

10. Hawkins KL, Montague BT, Rowan SE, Beum R, McLees MP, Johnson S, Gardner EM. Boosted darunavir and dolutegravir dual therapy among a cohort of highly treatment-experienced individuals. Antivir Ther. 2019. doi:10.3851/IMP3330.

11. Aoki M, Hayashi H, Yedidi RS, Martyr CD, Takamatsu Y, Aoki-Ogata H, Nakamura T, Nakata H, Das D, Yamagata Y, Ghosh AK, Mitsuya H. C-5-Modified Tetrahydropyrano-Tetrahydofuran-Derived Protease Inhibitors (PIs) Exert Potent Inhibition of the Replication of HIV-1 Variants Highly Resistant to Various PIs, including Darunavir. J Virol. 2015. 90:2180-2194.

12. Ide K, Aoki M, Amano M, Koh Y, Yedidi RS, Das D, Leschenko S, Chapsal B, Ghosh AK, Mitsuya H. Novel HIV-1 protease inhibitors (PIs) containing a bicyclic P2 functional moiety, tetrahydropyrano-tetrahydrofuran, that are potent against multi-PI-resistant HIV-1 variants. Antimicrob Agents Chemother. 2011. 55:1717-1727.

13. Tamiya S, Mardy S, Kavlick MF, Yoshimura K, Mistuya H. Amino acid insertions near Gag cleavage sites restore the otherwise compromised replication of human immunodeficiency virus type 1 variants resistant to protease inhibitors. J Virol. 2004; 78:12030-12040.

14. Yoshimura K, Kato R, Yusa K, Kavlick MF, Maroun V, Nguyen A, Mimoto T, Ueno T, Shintani M, Falloon J, Masur H, Hayashi H, Erickson J, Mitsuya H. JE-2147: a dipeptide protease inhibitor (PI) that potently inhibits multi-PI-resistant HIV-1. Proc Natl Acad Sci U S A. 1999; 96:8675-8680.

15. Aoki M, Danish ML, Aoki-Ogata H, Amano M, Ide K, Das D, Koh Y, Mitsuya H. Loss of the protease dimerization inhibition activity of tipranavir (TPV) and its association with the acquisition of resistance to TPV by HIV-1. J Virol. 2012; 86:13384-13396.

16. Aoki M, Venzon DJ, Koh Y, Aoki-Ogata H, Miyakawa T, Yoshimura K, Maeda K, Mitsuya H. Non-cleavage site gag mutations in amprenavir-resistant human immunodeficiency virus type 1 (HIV-1) predispose HIV1 to rapid acquisition of amprenavir resistance but delay development of resistance to other protease inhibitors. J Virol. 2009; 83:3059-3068.

17. Koh Y, Amano M, Towata T, Danish M, LeshchenkoYashchuk S, Das D, Nakayama M, Tojo Y, Ghosh AK, Mitsuya H. In vitro selection of highly darunavir-resistant and replication-competent HIV-1 variants by using a mixture of clinical HIV-1 isolates resistant to multiple conventional protease inhibitors. J Virol. 2010; 84:1196111969.

18. Hayashi H, Takamune N, Nirasawa T, Aoki M, Morishita Y, Das D, Koh Y, Ghosh AK, Misumi S, Mitsuya H.. Dimerization of HIV-1 protease occurs through two steps relating to the mechanism of protease dimerization inhibition by darunavir. Proc Natl Acad Sci U S A. 2014; 111:12234-12239. 
19. Aoki M, Hayashi H, Rao KV, et al. A novel central nervous system-penetrating protease inhibitor overcomes human immunodeficiency virus 1 resistance with unprecedented aM to pM potency. ELife. 2017; 6. doi: 10.7554/eLife.28020.

20. Yedidi RS, Maeda K, Fyvie WS, Steffey M, Davis DA, Palmer I, Aoki M, Kaufman JD, Stahl SJ, Garimella H, Das D, Wingfield PT, Ghosh AK, Mitsuya H. P2' benzene carboxylic acid moiety is associated with decrease in cellular uptake: evaluation of novel nonpeptidic HIV1 protease inhibitors containing P2 bis-tetrahydrofuran moiety. Antimicrob Agents Chemother. 2013. 57:49204927.

21. Desbois D, Roquebert B, Peytavin G, Damond F, Collin G, Benard A, Campa P, Matheron S, Chene G, Brun-Vezinet $\mathrm{F}$, Descamps D, French AHIVC. In vitro phenotypic susceptibility of human immunodeficiency virus type 2 clinical isolates to protease inhibitors. Antimicrob Agents Chemother. 2008; 52:1545-1548.

22. Aoki M, Das D, Hayashi H, Aoki-Ogata H, Takamatsu Y, Ghosh AK, Mitsuya H. Mechanism of Darunavir (DRV)'s high genetic barrier to HIV-1 resistance: A key V32I substitution in protease rarely occurs, but once it occurs, it predisposes HIV-1 to develop DRV resistance. MBio. 2018; 9. doi: 10.1128/mBio.02425-17.

23. Koh Y, Aoki M, Danish ML, Aoki-Ogata H, Amano M, Das D, Shafer RW, Ghosh AK, Mitsuya H. Loss of protease dimerization inhibition activity of darunavir is associated with the acquisition of resistance to darunavir by HIV-1. J Virol. 2011; 85:10079-10089.

24. Hong L, Hartsuck JA, Foundling S, Ermolieff J, Tang J. Active-site mobility in human immunodeficiency virus, type 1, protease as demonstrated by crystal structure of A28S mutant. Protein Sci. 1998; 7:300-305.

25. Yates PJ, Hazen R, St Clair M, Boone L, Tisdale M, Elston RC. In vitro development of resistance to human immunodeficiency virus protease inhibitor GW640385. Antimicrob Agents Chemother. 2006; 50:1092-1095.

26. Yoshimura K, Kato R, Kavlick MF, Nguyen A, Maroun V, Maeda K, Hussain KA, Ghosh AK, Gulnik SV, Erickson JW, Mitsuya H. A potent human immunodeficiency virus type 1 protease inhibitor, UIC-94003 (TMC-126), and selection of a novel (A28S) mutation in the protease active site. J Virol. 2002. 76:1349-1358.

27. Amano M, Koh Y, Das D, Li J, Leschenko S, Wang YF, Boross PI, Weber IT, Ghosh AK, Mitsuya H. A novel bistetrahydrofuranylurethane-containing nonpeptidic protease inhibitor (PI), GRL-98065, is potent against multiplePI-resistant human immunodeficiency virus in vitro. Antimicrob Agents Chemother. 2007; 51:2143-2155.

28. Wu TD, Schiffer CA, Gonzales MJ, Taylor J, Kantor R, Chou S, Israelski D, Zolopa AR, Fessel WJ, Shafer RW. Mutation patterns and structural correlates in human immunodeficiency virus type 1 protease following different protease inhibitor treatments. J Virol. 2003; 77:4836-4847.

29. Takamatsu Y, Aoki M, Bulut H, Das D, Amano M, Sheri VR, Kovari LC, Hayashi H, Delino NS, Ghosh AK, Mitsuya H. Novel protease inhibitors containing C-5-modified bis-tetrahydrofuranylurethane and aminobenzothiazole as P2 and P2' ligands that exert potent antiviral activity against highly multidrug-resistant HIV-1 with a high genetic barrier against the emergence of drug resistance. Antimicrob Agents Chemother. 2019; 63. doi: 10.1128/AAC.00372-19.

30. Delino NS, Aoki M, Hayashi H, Hattori SI, Chang SB, Takamatsu Y, Martyr CD, Das D, Ghosh AK, Mitsuya H. GRL-079, a novel HIV-1 protease inhibitor, is extremely potent against multidrug-resistant HIV-1 variants and has a high genetic barrier against the emergence of resistant variants. Antimicrob Agents Chemother. 2018; 62. doi: 10.1128/AAC.02060-17.

31. Hattori SI, Hayashi H, Bulut H, Rao KV, Nyalapatla PR, Hasegawa K, Aoki M, Ghosh AK, Mitsuya H. Halogen bond interactions of novel HIV-1 protease inhibitors (PI) (GRL-001-15 and GRL-003-15) with the flap of protease are critical for their potent activity against wildtype HIV-1 and multi-PI-resistant variants. Antimicrob Agents Chemother. 2019; 63. doi: 10.1128/AAC.0263518.

32. Dang Q, Chen J, Unutmaz D, Coffin JM, Pathak VK, Powell D, KewalRamani VN, Maldarelli F, Hu WS. Nonrandom HIV-1 infection and double infection via direct and cell-mediated pathways. Proc Natl Acad Sci U S A. 2004. 101:632-637.

33. Jung A, Maier R, Vartanian JP, Bocharov G, Jung V, Fischer U, Meese E, Wain-Hobson S, Meyerhans A. Recombination: multiply infected spleen cells in HIV patients. Nature. 2002; 418:144.

34. Blackard JT, Cohen DE, Mayer KH. Human immunodeficiency virus superinfection and recombination: current state of knowledge and potential clinical consequences. Clin Infect Dis. 2002; 34:11081114.

35. Moutouh L, Corbeil J, Richman DD. Recombination leads to the rapid emergence of HIV-1 dually resistant mutants under selective drug pressure. Proc Natl Acad Sci U S A. 1996; 93:6106-6111.

36. Yusa K, Kavlick MF, Kosalaraksa P, Mitsuya H. HIV1 acquires resistance to two classes of antiviral drugs through homologous recombination. Antiviral Res. 1997; $36: 179-189$

37. Koh Y, Matsumi S, Das D, Amano M, Davis DA, Li J, Leschenko S, Baldridge A, Shioda T, Yarchoan R, Ghosh AK, Mitsuya H. Potent inhibition of HIV-1 replication by novel non-peptidyl small molecule inhibitors of protease dimerization. J Biol Chem. 2007; 282:28709-28720.

38. De Meyer S, Azijn H, Surleraux D, Jochmans D, Tahri A, Pauwels R, Wigerinck P, de Bethune MP. TMC114, a novel human immunodeficiency virus type 1 protease inhibitor active against protease inhibitor-resistant viruses, including a broad range of clinical isolates. Antimicrob Agents Chemother. 2005; 49:2314-2321.

Received September 30, 2019; Revised October 9, 2019; Accepted October 15, 2019.

*Address correspondence to:

Hiroaki Mitsuya, The Experimental Retrovirology Section, HIV \& AIDS Malignancy Branch, National Cancer Institute, National Institutes of Health, 9000 Rockville Pike, Building 10, Room 5A11, Bethesda, Maryland, USA.

E-mail: mitsuyah@nih.gov 